\title{
TRATAMENTO DE BIOGÁS PARA ATENUAR O EFEITO CORROSIVO
}

\author{
F. G. M. PORTO ${ }^{1}$, M. L. BEGNINI ${ }^{1}$ e J. R. D. FINZER ${ }^{1}$ \\ ${ }^{1}$ Universidade de Uberaba, Curso de Engenharia Química \\ fabriciomenezesporto@hotmail.com
}

\begin{abstract}
RESUMO - A corrosão pode ser definida como a deterioração de um material, por ação física, química ou eletroquímica. Conhecendo os meios agressivos e suas características, podem ser desenvolvidos métodos eficazes para evitar a corrosão, sendo uma delas a absorção do agente corrosivo. Para aplicar a absorção em uma situação prática, selecionou-se o caso do ácido sulfídrico. Sendo esse um agente altamente oxidante, a concentração em excesso desse composto em correntes de processos industriais pode acarretar em um agravamento da corrosão de equipamentos. Uma das formas de diminuir esse problema é realizar a técnica de absorção de gases, de modo que a concentração do ácido seja consideravelmente diminuída. Foi realizado estudo de absorção de ácido sulfídrico utilizando uma solução aquosa de hidróxido de sódio a $5 \%$. O equipamento projetado consistiu em uma coluna de recheio cilíndrica, equipada com uma entrada de gás e um distribuidor no fundo, que também suporta o recheio, além de uma entrada de líquido e um distribuidor no topo. O gás, após absorção de solutos, é descarregado no topo da coluna, e o líquido, no fundo, contendo o soluto que foi absorvido. No estudo, a vazão de gás foi de $12,5 \mathrm{~m}^{3} / \mathrm{h}$ com $3 \% \mathrm{em}$ mol, de ácido sulfídrico. A coluna de absorção foi preenchida com recheio de anéis Raschig de 1,5 polegadas. A partir dos cálculos realizados, especificou-se uma coluna de absorção com 0,10 $\mathrm{m}$ de diâmetro e $3 \mathrm{~m}$ de altura do recheio, e com perda de pressão de $0,5 \mathrm{~cm}$ água/m.
\end{abstract}

\section{INTRODUÇÃO}

A corrosão pode ser definida como a deterioração de um material, geralmente metálico, por ação física, química ou eletroquímica do meio ambiente, aliada ou não, a esforços mecânicos. Sendo a corrosão, em geral, um processo espontâneo, ela está constantemente transformando os materiais metálicos de modo que a durabilidade e desempenho dos mesmos deixem de satisfazer os fins a que se destinam. A deterioração causada pela interação físicoquímica entre o material e o meio em que este se encontra, leva a alterações prejudiciais e indesejáveis, como: desgaste, transformações químicas ou modificações estruturais, tornando o material inadequado para o uso.

O estudo dos processos de corrosão se encontra em grande ascendência, já que muitas falhas dos materiais têm sido atribuídas a este fator. Esse fenômeno pode ser acelerado com o aumento da temperatura, da pressão e de altas concentrações do meio corrosivo. Conhecendo os meios agressivos e suas características responsáveis pela deterioração dos materiais, podem ser desenvolvidos métodos eficazes para combater à corrosão, que devem ser escolhidos 
dependendo da natureza do material que será protegido e do eletrólito (meio corrosivo). $\mathrm{O}$ custo e o tempo necessários para o emprego do método em questão devem ser considerados (Frauches-Santos et al., 2013).

Uma das formas de evitar a corrosão consiste na técnica de absorção do agente corrosivo. As moléculas do gás corrosivo são difundidas em um líquido. Essa operação pode ser classificada em dois grupos principais: um em que apenas processos físicos ocorrem e outro em que ocorrem reações químicas (Leite et al., 2005).

A absorção com reação química tem vasta aplicação industrial, principalmente para a remoção de gases ácidos, misturas inertes e hidrocarbonetos em correntes de gases. Quando utilizada, a reação química aumenta a eficiência de transferência de massa, devido a presença dos reagentes. Além disso, a manipulação dos parâmetros de operação (temperatura, pressão, vazões) influencia diretamente nas taxas de reação. Sendo o ácido sulfídrico $\left(\mathrm{H}_{2} \mathrm{~S}\right)$ um agente altamente oxidante, a presença em excesso desse composto em processos industriais pode acarretar em um agravamento da corrosão de determinados equipamentos. Uma das formas de atenuar esse problema é realizar a técnica de absorção de gás, de modo que a concentração de $\mathrm{H}_{2} \mathrm{~S}$ seja inferior à padronizada. Para isso, pode ser utilizada uma solução cáustica capaz de reagir com o ácido através de uma reação ácido-base, produzindo produtos solúveis na corrente líquida. Esse procedimento se mostra bastante eficiente, sendo, amplamente utilizado em indústrias que processam gases contendo gás sulfídrico. (Richardson et al., 2002).

Este trabalho tem o objetivo de especificar uma coluna de absorção de gases para separação do ácido sulfídrico contido em biogás usando uma solução cáustica no processo.

\section{SISTEMÁTICA PARA DIMENSIONAMENTO DA COLUNA DE ABSORÇÃO}

O equipamento utilizado na absorção de gases consiste de uma coluna cilíndrica ou torre, equipados com uma entrada de gás e um distribuidor no fundo, o qual também suporta o recheio, além de uma entrada de líquido e um distribuidor no topo. O gás, após absorção de solutos, é descarregado no topo da coluna, e o líquido contendo o soluto que foi absorvido, é descarregado no fundo da coluna. O equipamento é denominado Torre ou Coluna de Recheio (Tower Packing), conforme mostra a Figura 1.

Em colunas recheadas, o ponto de inundação (flooding point) corresponde à condição em que o líquido ocupa toda área da seção transversal da coluna. O fluxo de gás deve ser otimizado, contudo se aproxima de 50\% da correspondente à condição de inundação (McCabe et al., 2004).

A Figura 2 possibilita obter a perda de pressão no recheio em polegada de água/ft de recheio; $\mathrm{u}_{0}$ é a velocidade superficial do gás em $\mathrm{ft} / \mathrm{s} ; v$ é a viscosidade do líquido em centistoke; Gx e Gy correspondem aos fluxos de líquido e de gás, respectivamente, e são quantificados em $\mathrm{kg} / \mathrm{m}^{2} . \mathrm{s}$; $\rho_{\mathrm{x}}$ e $\rho_{\mathrm{y}}$ são as densidades $\mathrm{em} \mathrm{kg} / \mathrm{m}^{3}$, é Cs é quantificado pela Equação 1 , sendo $u_{0}$ a velocidade superficial do gás na coluna, $F_{P}$ é um fator de perda de pressão que depende da geometria e caracterização do recheio (McCabe et al., 2004). 


$$
C_{S}=u_{0} \cdot \sqrt{\frac{\rho_{y}}{\rho_{x}-\rho_{y}}}
$$

A área da seção transversal da coluna pode ser calculada com a Equação 2, sendo $S$ a área da seção transversal da coluna; $W$ e $G_{y}$ operação, a taxa mássica e o fluxo de gás, respectivamente.

$$
S=\frac{W}{G_{y \text { operação }}}
$$

Figura 1 - Exemplo de torre recheada (tower packing).

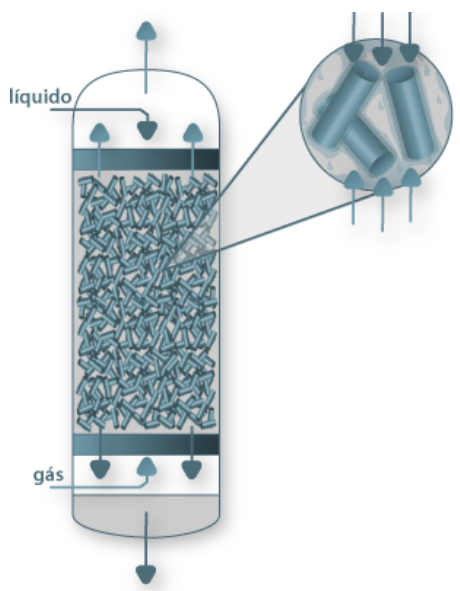

Figura 2 - Correlação generalizada para inundação e perda de pressão em colunas.

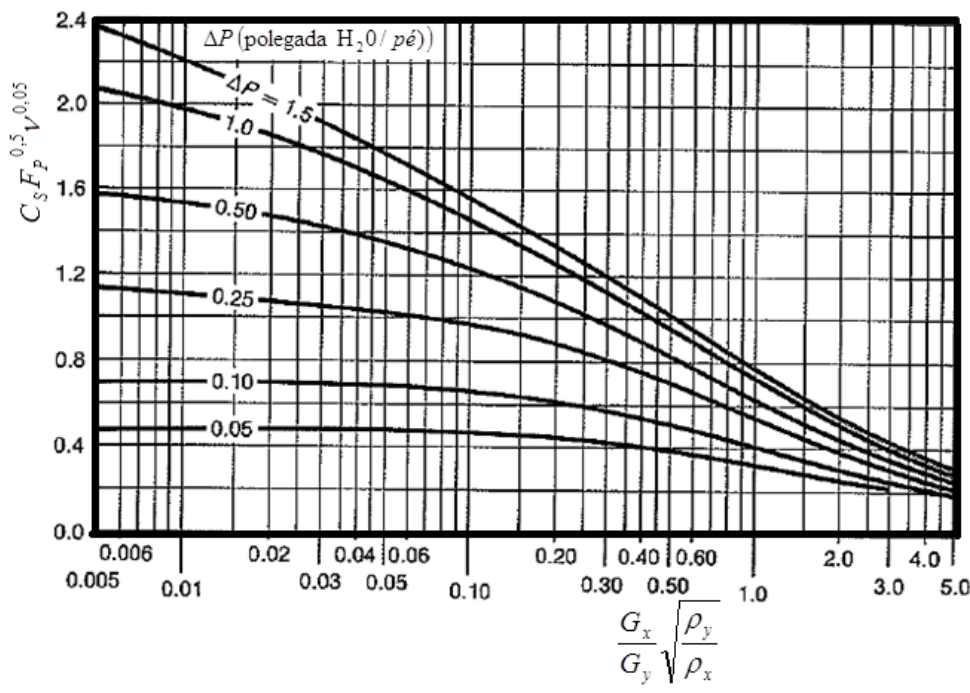

A altura do recheio da coluna de absorção, $Z_{t}$, é calculada com a Equação 3.

$$
Z_{t}=N_{0 y} \cdot H_{0 y}
$$


Sendo: $\mathrm{H}_{0 \mathrm{y}}$ a altura de uma unidade de transferência, quantificado pela Figura 3 e $\mathrm{N}_{0 \mathrm{y}} \mathrm{o}$ número de unidades de transferência, sendo quantificado pela Equação 4.

$$
N_{0 y}=\int_{y_{\text {entrada }}}^{\mathrm{y}_{\text {saida }}} \frac{\mathrm{dy}}{\mathrm{y}-\mathrm{y}^{*}}
$$

Sendo: $y$ e $y^{*}$ frações molares do soluto na fase gasosa e a de equilíbrio com o líquido de absorção.

Figura 3 - Altura da unidade de transferência (Norman, 1962).

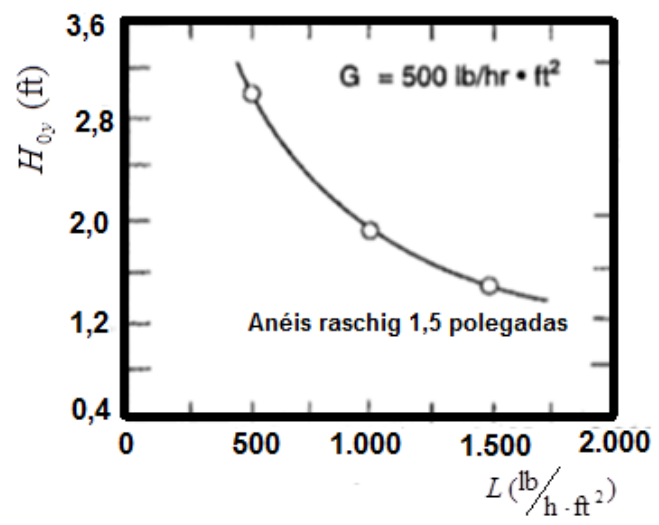

A perda de pressão (polegada de água/pé altura recheio) na condição de inundação é obtida pela Equação 5, sendo $F_{P}=95$ para anéis Raschig de 1,5 polegadas (McCabe et al., 2004).

$$
\Delta P_{\text {inundas̆a }}=0,115 \cdot F_{P}^{0,7}
$$

\section{SISTEMA DE PRODUÇÃO DE ÁCIDO SULFÍDRICO}

Este estudo faz parte do sistema de produção de gás em biodigestor instalado nas dependências da Universidade de Uberaba - MG. O biodigestor produz 12,5 $\mathrm{m}^{3} / \mathrm{h}$ de gás com $3 \%$ mol $(3,5 \% \mathrm{~m} / \mathrm{m})$, em média, de $\mathrm{H}_{2} \mathrm{~S}$, medido na temperatura de $25^{\circ} \mathrm{C}$ e pressão barométrica local. Deve-se absorver 99\% do ácido sulfídrico.

\section{RESULTADOS E DISCUSSÃO}

\subsection{Diâmetro da coluna de absorção}

Para o dimensionamento da coluna de absorção são requeridos parâmetros estequiométricos na utilização das equações de projeto. A massa molecular média do gás de admissão calculado com o dado de $3 \%$ em mol de ácido sulfídrico consiste em $29,15 \mathrm{~kg} / \mathrm{kmol}$. A densidade do gás medida nas condições operacionais, usando a lei dos gases, $1,192 \mathrm{~kg} / \mathrm{m}^{3}$. 
A taxa de escoamento do gás na coluna pode ser quantificada:

$$
W=12,5 \mathrm{~m}^{3} / h \cdot 1,192 \mathrm{~kg} / \mathrm{m}^{3}=14,9 \mathrm{~kg} / \mathrm{h}(32,82 \mathrm{lb} / \mathrm{h})
$$

O dimensionamento da coluna de absorção foi realizado por método iterativo. O ponto de partida foi o fluxo de gás de operação de $500 \mathrm{lb} / \mathrm{h} \cdot \mathrm{ft}^{2}$, o que possibilita especificar o diâmetro da coluna usando a Equação 2.

$$
S=\frac{W}{G_{y \text { operação }}}=\frac{32,82 \mathrm{lb} / \mathrm{h}}{500 \mathrm{lb} / \mathrm{h} \cdot \mathrm{ft}^{2}}=0,066 \mathrm{ft}^{2}
$$

Como a coluna é cilíndrica calcula-se o diâmetro: $\mathrm{D}=0,300 \mathrm{ft}(0,100 \mathrm{~m})$.

\subsection{Fluxo de solução alcalina de absorção com reação química}

A quantidade de ácido sulfídrico na alimentação da coluna é obtida do produto da taxa mássica de gás a ser tratado pela fração mássica da corrente $(0,035)$ consistindo em $0,52 \mathrm{~kg} / \mathrm{h}$ e a quantidade de hidróxido de sódio é calculada pela estequiometria da reação.

$$
2 \mathrm{NaOH}+\mathrm{H}_{2} \mathrm{~S} \rightarrow \mathrm{Na}_{2} \mathrm{~S}+2 \mathrm{H}_{2} \mathrm{O}
$$

Como a proporção molar é de dois para um, a taxa teórica de hidróxido de sódio é de $1,22 \mathrm{~kg} / \mathrm{h}$.

Contudo, em gases contendo dióxido de carbono ocorre precipitação de bicarbonato de sódio quando o $\mathrm{pH}$ é baixo, e sulfeto de sódio e carbonato de sódio em $\mathrm{pH}$ alto, porém as reações são lentas (Mamrosh et al., 2008). Para minimizar esse efeito selecionou-se 20\% a mais de hidróxido de sódio. Assim, a taxa de hidróxido de sódio deverá ser de 1,47 kg/h e a taxa da solução $29,4 \mathrm{~kg} / \mathrm{h}(64,8 \mathrm{lb} / \mathrm{h})$ a $5 \%$ em massa. A densidade da solução é igual a 1.054 $\mathrm{kg} / \mathrm{m}^{3}$, o que possibilita o cálculo da vazão em $0,028 \mathrm{~m}^{3} / \mathrm{h}$. O fluxo de solução pode ser calculado:

$$
G_{x}=\frac{64,8 \mathrm{lb} / \mathrm{h}}{\pi \frac{0,33^{2}}{4} f t^{2}}=758 \mathrm{lb} / \mathrm{h} \cdot \mathrm{ft}^{2}
$$

\subsection{Altura da coluna}

A reação do ácido sulfídrico com o hidróxido de sódio é bastante rápida e o valor de equilíbrio com uma solução tende a zero o que facilita o tratamento matemático (Mamrosh, et al., 2008). A Equação 4 possibilita o cálculo de $N_{O y}$, simplificada pelas considerações efetuadas.

$$
N_{0 y}=\int_{y_{\text {entrada }}}^{y_{\text {saida }}} \frac{\mathrm{dy}}{\mathrm{y}}=\ln y_{0,03}^{0,0003}=\ln 0,03-\ln 0,0003=4,61
$$

A altura da unidade de transferência é obtida da Figura 3, obtendo-se: $H_{O y}=2,2 \mathrm{ft}$ e usando a Equação 3, a altura da coluna deve ser de $10 \mathrm{ft}$ (3 m). 


\subsection{Perda de pressão na coluna}

Com os fluxos Gx e Gy, a viscosidade da solução $v=1,2 c S$, e a velocidade superficial do gás $0,44 \mathrm{~m} / \mathrm{s}(1,45 \mathrm{ft} / \mathrm{s})$, calcula-se $C_{S}=0,05$; o valor da abscissa é quantificado em 0,052 e:

$$
C_{S} F_{P}{ }^{0,5} v^{0,05}=0,05 \cdot 95^{0,5} \cdot 1,2^{0,05}=0,5
$$

Utilizando a Figura 2, obtém-se: $\Delta P=0,06$ polegadas água/pé de recheio $(0,5 \mathrm{~cm}$ água/m). A Equação 5 possibilita quantificar a perda de pressão na condição de inundação:

$\Delta P_{\text {inundas̆ão }}=0,115 \cdot 95^{0,7}=2,8$ polegada de água/pé altura recheio

A perda de pressão na operação é $2,1 \%$ da perda de pressão na inundação, o que consiste em indicação de funcionamento adequado da coluna e pode-se reduzir a dimensão do recheio para melhorar a eficiência da transferência de massa. Os fatores $F_{P}$ para anéis Raschig de 0,5 e de 1,0 polegadas são 580 e 155, respectivamente (McCabe et al., 2004). A Equação 5 possibilita o cálculo das perdas de pressão na condição de inundação para os dois anéis de 0,5 e de 1,0 polegadas igual 9,9 e 3,9 polegada de água/pé altura recheio, respectivamente.

\section{CONCLUSÕES}

O estudo realizado na especificação de uma coluna de absorção de gases para processar $12,5 \mathrm{~m}^{3} / \mathrm{h}$ de biogás a $3 \% \mathrm{~mol}$ de $\mathrm{H}_{2} \mathrm{~S}$ resultou em altura e diâmetro $3 \mathrm{~m} \mathrm{e} 0,1 \mathrm{~m}$, respectivamente, operando com perda de pressão de $0,5 \mathrm{~cm}$ água $/ \mathrm{m}$. Isso indica que pode ser diminuído o tamanho dos anéis Raschig para melhorar a eficiência da coluna de absorção.

\section{REFERÊNCIAS}

FRAUCHES-SANTOS,C; ALBUQUERQUE, M. A.; OlIVEIRA, M. C. C.; ECHEVARRIA, A. A. A Corrosão e os Agentes Anticorrosivos. Rev. Virtual Quim., v. 6, p. 293-309, 2014.

LEITE, A. B.; BERTOLI, S. L.; BARROS, A. A. C. Absorção Química de dióxido de nitrogênio. Eng. Sanit. Ambient. v.10, 2005.

MAMROSH, D.; BEITLER, C.; FISHER, K. Consider improved scrubbing designs for acid gases: Better application of process chemistry enables efficient sulfur abatement. Hydrocarbon Processing. p. 69-74, 2008.

McCABE, W. L.; SMITH, J. C.;HARRIOT, P. Unit operations of chemical engineering. 6. ed. Boston: McGraw Hill, 2005.

NORMAN, W.S. Absorption, distillation and cooling towers. London: Longmans. 1962.

RICHARDSON, J., COULSON, J. Chemical engineering design. Boston: ButterworthHeinemann. 2002. 